ANNALES

POLONICI MATHEMATICI

$91.2-3(2007)$

\title{
Continuous pluriharmonic boundary values
}

\author{
by Per Ånag (Sundsvall) and Rafą Czyż (Kraków)
}

\begin{abstract}
Let $D_{j}$ be a bounded hyperconvex domain in $\mathbb{C}^{n_{j}}$ and set $D=D_{1} \times$ $\cdots \times D_{s}, j=1, \ldots, s, s \geq 3$. Also let $\mathbb{G}_{n}$ be the symmetrized polydisc in $\mathbb{C}^{n}, n \geq 3$. We characterize those real-valued continuous functions defined on the boundary of $D$ or $\mathbb{G}_{n}$ which can be extended to the inside to a pluriharmonic function. As an application a complete characterization of the compliant functions is obtained.
\end{abstract}

1. Introduction. Let $\Omega \subseteq \mathbb{C}^{n}$ be a bounded domain. The overdetermined system of equations which defines pluriharmonic functions in $\Omega$,

$$
\frac{\partial^{2} u}{\partial z_{j} \partial \bar{z}_{k}}=0, \quad j, k=1, \ldots, n,
$$

has been considered by mathematicians for more than a century (see e.g. [24]). It is well-known that for a continuous function $f: \partial \Omega \rightarrow \mathbb{R}$ there does not always exist a pluriharmonic function $u$ which is continuous on $\bar{\Omega}$ such that $\left.u\right|_{\partial \Omega}=f$. This Dirichlet problem has been extensively studied for the case of smoothly bounded domains, like the unit ball, strictly pseudoconvex domains or the unit polydisc. We refer to [5] and [17] for details and references. We would especially like to draw attention to the article [4] and the more recently published [6], [14], [22] and [23].

We will prove a complete characterization of the Dirichlet problem for pluriharmonic functions defined on bounded hyperconvex product domains and on the symmetrized polydisc in $\mathbb{C}^{n}, n \geq 3$ (see e.g. Section 5 for the definition of the symmetrized polydisc). Our methods rely purely on potential theory. For an introduction to classical and pluripotential theory the monographs [3] and [21] are recommended. The aim of this article is to prove the following theorem:

2000 Mathematics Subject Classification: Primary 31C10; Secondary 32U15.

Key words and phrases: analytic disc, compliant function, Dirichlet problem, Jensen measure, pluriharmonic function, symmetrized polydisc.

The second-named author was partially supported by KBN grant 1 P03A 03726 . 
Theorem A. Let $D_{j}$ be a bounded hyperconvex domain in $\mathbb{C}^{n_{j}}, n_{j} \geq 1$. Set $D=D_{1} \times \cdots \times D_{s}, j=1, \ldots, s, s \geq 3$, and let $\mathbb{G}_{n}$ be the symmetrized polydisc in $\mathbb{C}^{n}, n \geq 3$. If $\Omega \in\left\{D, \mathbb{G}_{n}\right\}$ and $f: \partial \Omega \rightarrow \mathbb{R}$ is a continuous function, then the following assertions are equivalent:

(1) there exists a function $u$ which is pluriharmonic on $\Omega$, continuous on $\bar{\Omega}$ and $\left.u\right|_{\partial \Omega}=f$,

(2) the function $f$ is pluriharmonic on $\partial \Omega$ (see Definitions 3.1 and 5.1),

(3) the Perron-Bremermann envelope $P B_{f}$ is pluriharmonic on $\Omega$, i.e.,

$$
P B_{-f}=-P B_{f},
$$

(4) for every $z_{0} \in \partial \Omega$ and every Jensen measure $\mu$ with barycenter $z_{0}$

$$
f\left(z_{0}\right)=\int_{\partial \Omega} f d \mu .
$$

The proof of Theorem A is divided into two parts, Theorem 3.3 for the case when $\Omega$ is a hyperconvex product domain $D$ and Theorem 5.4 for $\Omega=\mathbb{G}_{n}$. In Section 4 we will show, for $s=n \geq 3$, that the conditions in Theorem A hold if and only if $f$ is subharmonic on every analytic disc $d$ embedded in $\partial D$, i.e., for every injective holomorphic function $d: \mathbb{D} \rightarrow \partial D$ the function $f \circ d$ is subharmonic on the unit disc $\mathbb{D} \subseteq \mathbb{C}$ (Theorem 4.2; see also Theorem 4.4). Theorem 4.2 will be used in the proof of Theorem A for $\Omega=\mathbb{G}_{n}$. If $n=2$ the implication $(2) \Rightarrow(1)$ is, in general, not true (Example 3.4 and Section 5). But (2) is equivalent to (4), (1) is equivalent to (3), and (1) always implies (2). As an application of Theorem A, we obtain a full characterization of the so called compliant functions (Corollary 3.6 and Theorem 5.4). They first appeared in [10] where the so-called Cegrell classes with boundary values given by a continuous function were introduced. For further information about the Cegrell classes see e.g. [11] and the references therein.

The authors would like to thank Jonas Andersson, Sławomir Kołodziej, Evgeny A. Poletsky and Frank Wikström for their generous help and encouragement.

2. Definitions and basic facts. Throughout this article, $D_{j}$ is a bounded domain in $\mathbb{C}^{n_{j}}$ and we set

$$
D=D_{1} \times \cdots \times D_{s},
$$

where $j=1, \ldots, s$. Let $n=n_{1}+\cdots+n_{s}$. Then $D$ is a bounded domain in $\mathbb{C}^{n}$ with the $(2 n-1)$-real dimensional boundary $\partial D$ given by

$$
\partial D=\bigcup_{j=1}^{s} \bar{D}_{1} \times \cdots \times \bar{D}_{j-1} \times \partial D_{j} \times \bar{D}_{j+1} \times \cdots \times \bar{D}_{s} .
$$


The boundary $\partial D$ can also be expressed as

$$
\begin{aligned}
\bigcup_{\substack{1 \leq j_{1}<\cdots<j_{k} \leq s \\
k \in\{1, \ldots, s\}}} D_{1} \times \cdots \times D_{j_{1}-1} \times \partial D_{j_{1}} \times D_{j_{1}+1} \times \cdots \\
\quad \times D_{j_{k}-1} \times \partial D_{j_{k}} \times D_{j_{k}+1} \times \cdots \times D_{s}=: \bigcup_{\substack{|J|=k \\
1 \leq k \leq s}} \Lambda^{J},
\end{aligned}
$$

where $J$ is the increasing multi-index, $1 \leq j_{1}<\cdots<j_{k} \leq s$, of length $k$; also we will use the notation $\widehat{\Lambda}^{J}$ for the open set in $\mathbb{C}^{n-n_{J}}$ given by $D_{1} \times \cdots \times D_{j_{1}-1} \times \widehat{\partial D_{j_{1}}} \times D_{j_{1}+1} \times \cdots \times D_{j_{k}-1} \times \widehat{\partial D_{j_{k}}} \times D_{j_{k}+1} \times \cdots \times D_{s}$, where $n_{J}=n_{j_{1}}+\cdots+n_{j_{k}}$. The distinguished boundary $\partial D^{+}$of $D$ is defined by $\partial D^{+}=\partial D_{1} \times \cdots \times \partial D_{s}$, so $\partial D^{+}=\Lambda^{J}$ when $J=\{1, \ldots, s\}$.

Recall that a bounded domain $\Omega \subseteq \mathbb{C}^{n}$ is called hyperconvex if there exists a plurisubharmonic exhaustion function $\varphi: \Omega \rightarrow(-\infty, 0)$ such that the closure of $\{z \in \Omega: \varphi(z)<c\}$ is compact in $\Omega$ for every $c \in(-\infty, 0)$. A bounded hyperconvex domain $\Omega$, viewed as a domain in $\mathbb{R}^{2 n}$, is always regular with respect to the Dirichlet problem for the Laplace operator. The Hartogs triangle, $\left\{\left(z_{1}, z_{2}\right) \in \mathbb{C}^{2}:\left|z_{1}\right|<\left|z_{2}\right|<1\right\}$, shows that not every regular, bounded pseudoconvex domain is hyperconvex.

Proposition 2.1. Let $D_{j}$ be a bounded domain in $\mathbb{C}^{n_{j}}, j=1, \ldots, s$, and set $n=n_{1}+\cdots+n_{s}$. Then $D=D_{1} \times \cdots \times D_{s} \subseteq \mathbb{C}^{n}$ is hyperconvex if and only if each $D_{j}$ is hyperconvex in $\mathbb{C}^{n_{j}}$.

Proof. Assume that $D$ is hyperconvex in $\mathbb{C}^{n}$, i.e., there exists a plurisubharmonic exhaustion function $\varphi$ for $D$. Fix $\left(z_{1}^{0}, \ldots, \widehat{z}_{j}, \ldots, z_{s}^{0}\right) \in D_{1} \times \ldots \times$ $\widehat{D}_{j} \times \cdots \times D_{s}$. Then

$$
\varphi_{j}(\zeta)=\varphi\left(z_{1}^{0}, \ldots, z_{j-1}^{0}, \zeta, z_{j+1}^{0}, \ldots, z_{s}^{0}\right)
$$

is an exhaustion function for $D_{j}$. Hence, $D_{j}$ is hyperconvex in $\mathbb{C}^{n_{j}}$. For the converse, assume that every $D_{j}$ is hyperconvex in $\mathbb{C}^{n_{j}}$ and $\varphi_{j}$ is an exhaustion function for $D_{j}$. Define $\varphi\left(\zeta_{1}, \ldots, \zeta_{s}\right)=\max \left\{\varphi_{1}\left(\zeta_{1}\right), \ldots, \varphi_{s}\left(\zeta_{s}\right)\right\}$ for $\left(\zeta_{1}, \ldots, \zeta_{s}\right) \in D$. Then $\varphi$ is a plurisubharmonic exhaustion function for $D$ and thus $D$ is hyperconvex in $\mathbb{C}^{n}$.

Definition 2.2. Let $\Omega \subseteq \mathbb{C}^{n}$ be a bounded domain and let $\mu$ be a non-negative, regular Borel measure on $\bar{\Omega}$. Then $\mu$ is a Jensen measure with barycenter at $z \in \bar{\Omega}$ if

$$
u(z) \leq \int_{\bar{\Omega}} u d \mu
$$

for every continuous function $u: \bar{\Omega} \rightarrow[-\infty, \infty)$, not identically $-\infty$, such that $u \in \mathcal{P S H}(\Omega)$. Here $\mathcal{P S H}(\Omega)$ is the class of all plurisubharmonic func- 
tions defined on $\Omega$. The set of all Jensen measures with barycenter at $z$ will be denoted by $\mathcal{J}_{z}$.

Definition 2.2 differs slightly from the classical definition of Jensen measure, since it allows the measure to have support in $\bar{\Omega}$, and also since it embraces Jensen measures for boundary points. If $\Omega$ is a bounded hyperconvex domain and $\mu \in \mathcal{J}_{z}, z \in \partial \Omega$, then supp $\mu \subseteq \partial \Omega$ (Theorem 3.4 in [30]). The Perron-Bremermann envelope for a function $f: \partial \Omega \rightarrow \mathbb{R}$ is defined by

$$
P B_{f}(z)=\sup \left\{w(z): w \in \mathcal{P} \mathcal{S H}(\Omega), \limsup _{\zeta \rightarrow \xi, \zeta \in \Omega} w(\zeta) \leq f(\xi) \forall \xi \in \partial \Omega\right\} .
$$

THEOREM 2.3. Let $\Omega \subseteq \mathbb{C}^{n}$ be a bounded domain and let $f: \partial \Omega \rightarrow \mathbb{R}$ be a continuous function. If

$$
\liminf _{z \rightarrow \xi, z \in \Omega} P B_{f}(z)=\limsup _{z \rightarrow \xi, z \in \Omega} P B_{f}(z)=f(\xi)
$$

for every $\xi \in \partial \Omega$, then $P B_{f} \in C(\bar{\Omega})$.

Proof. See [29].

THEOREM 2.4. Assume that $\Omega \subseteq \mathbb{C}^{n}$ is a bounded domain and that $f: \partial \Omega \rightarrow \mathbb{R}$ is a continuous function. The following assertions are then equivalent:

(1) for every $\xi \in \partial \Omega$,

$$
\lim _{z \rightarrow \xi, z \in \Omega}\left(P B_{f}+P B_{-f}\right)(z)=0,
$$

(2) there exists a continuous function $F: \bar{\Omega} \rightarrow \mathbb{R}$ such that for every $z_{0} \in \partial \Omega$ and every $\mu \in \mathcal{J}_{z_{0}}$,

$$
f\left(z_{0}\right)=\int_{\bar{\Omega}} F d \mu
$$

(3) the envelopes $P B_{f}$ and $P B_{-f}$ belong to $C(\bar{\Omega})$; moreover, for every $z_{0} \in \partial \Omega$ and every $\mu \in \mathcal{J}_{z_{0}}$,

$$
f\left(z_{0}\right)=\int_{\bar{\Omega}} P B_{f} d \mu \quad \text { and } \quad-f\left(z_{0}\right)=\int_{\bar{\Omega}} P B_{-f} d \mu .
$$

Proof. (3) $\Rightarrow(2)$ : Take $F=P B_{f}$.

$(2) \Rightarrow(1)$ : Lemma 3.3 in [30] implies that there exist $u, v \in \mathcal{P S H}(\Omega) \cap$ $C(\bar{\Omega})$ such that

$$
\lim _{z \rightarrow \zeta, z \in \Omega} u(z)=f(\zeta) \text { and } \lim _{z \rightarrow \xi, z \in \Omega} v(z)=-f(\xi)
$$

for all $\zeta, \xi \in \partial \Omega$, hence $(2.2)$ is satisfied.

$(1) \Rightarrow(3)$ : First we will prove that assumption (2.2) implies that

$$
\lim _{z \rightarrow \zeta, z \in \Omega} P B_{f}(z)=f(\zeta) \text { and } \lim _{z \rightarrow \xi, z \in \Omega} P B_{-f}(z)=-f(\xi)
$$


for all $\zeta, \xi \in \partial \Omega$. Assume now that this is not the case, for example there exists $\xi \in \partial \Omega$ such that $\lim \sup _{z \rightarrow \xi} P B_{f}(z)<f(\xi)$. This yields

$$
\begin{aligned}
0 & =\lim _{z \rightarrow \xi, z \in \Omega}\left(P B_{f}+P B_{-f}\right)(z)=\limsup _{z \rightarrow \xi, z \in \Omega}\left(P B_{f}+P B_{-f}\right)(z) \\
& \leq \limsup _{z \rightarrow \xi, z \in \Omega} P B_{f}(z)+\limsup _{z \rightarrow \xi, z \in \Omega} P B_{-f}(z) \\
& <f(\xi)-f(\xi)=0,
\end{aligned}
$$

a contradiction, hence limsup $P B_{f}=f$ and limsup $P B_{-f}=-f$ on $\partial \Omega$. Assume now that there exists $\zeta \in \partial \Omega$ such that $\liminf _{z \rightarrow \zeta} P B_{f}(z)<f(\zeta)$. Then there exists a sequence $\left[z_{j}\right]$ in $\Omega$ which converges to $\zeta$ such that $\lim _{j \rightarrow \infty} P B_{f}\left(z_{j}\right)<f(\zeta)$, hence

$$
\begin{aligned}
0 & =\lim _{j \rightarrow \infty}\left(P B_{f}+P B_{-f}\right)\left(z_{j}\right)=\liminf _{j \rightarrow \infty}\left(P B_{f}+P B_{-f}\right)\left(z_{j}\right) \\
& =\lim _{j \rightarrow \infty} P B_{f}\left(z_{j}\right)+\liminf _{j \rightarrow \infty} P B_{-f}\left(z_{j}\right)<f(\zeta)-f(\zeta)=0,
\end{aligned}
$$

a contradiction once more. Now it follows by Theorem 2.3 that $P B_{f}, P B_{-f} \in$ $C(\bar{\Omega})$. Fix $z_{0} \in \partial \Omega$ and take $\mu \in \mathcal{J}_{z_{0}}$; then

$$
f\left(z_{0}\right)=P B_{f}\left(z_{0}\right) \leq \int_{\bar{\Omega}} P B_{f} d \mu .
$$

Thus

$$
f\left(z_{0}\right) \leq \inf \left\{\int_{\bar{\Omega}} P B_{f} d \mu: \mu \in \mathcal{J}_{z_{0}}\right\}
$$

Setting $\mu=\delta_{z_{0}}$ shows that

$$
f\left(z_{0}\right)=\inf \left\{\int_{\bar{\Omega}} P B_{f} d \mu: \mu \in \mathcal{J}_{z_{0}}\right\} .
$$

In a similar manner the corresponding formula can be obtained for $-f$ and therefore

$$
\sup \left\{\int_{\bar{\Omega}}-P B_{-f} d \mu: \mu \in \mathcal{J}_{z_{0}}\right\}=-\inf \left\{\int_{\bar{\Omega}} P B_{-f} d \mu: \mu \in \mathcal{J}_{z_{0}}\right\}=f\left(z_{0}\right) .
$$

The maximum principle for plurisubharmonic functions and assumption (2.2) yield

$$
\inf \left\{\int_{\bar{\Omega}} P B_{f} d \mu: \mu \in \mathcal{J}_{z_{0}}\right\}=f\left(z_{0}\right) \geq \sup \left\{\int_{\bar{\Omega}} P B_{f} d \mu: \mu \in \mathcal{J}_{z_{0}}\right\} .
$$

Thus, for every $z_{0} \in \partial \Omega$ and every $\mu \in \mathcal{J}_{z_{0}}$ we have

$$
f\left(z_{0}\right)=\int_{\bar{\Omega}} P B_{f} d \mu .
$$

With the same methods the corresponding result can be proved for $-f$, hence (2.3) is true and the proof is complete. 
REMARK. Let $\Omega \subseteq \mathbb{C}^{n}$ be a bounded domain. The following are then equivalent:

(1) $\Omega$ is B-regular,

(2) property (2.2) holds for every continuous function $f: \partial \Omega \rightarrow \mathbb{R}$,

(3) $\mathcal{J}_{z_{0}}=\left\{\delta_{z_{0}}\right\}$ for every $z_{0} \in \partial \Omega$, where $\delta_{z_{0}}$ denotes the Dirac measure at $z_{0}$.

(See e.g. Corollary 3.8 in [30] for the equivalence between (1) and (3)).

3. Pluriharmonic boundary values on hyperconvex product domains. Let $\Omega$ be an open set in $\mathbb{C}^{n}$. A function $u: \Omega \rightarrow \mathbb{R}$ is said to be pluriharmonic on $\Omega$ if $u$ and $-u$ are plurisubharmonic on $\Omega$. Let $\mathcal{P H}(\Omega)$ denote the class of all pluriharmonic functions on $\Omega$. Then $\mathcal{P H}(\Omega) \subseteq \mathcal{P} \mathcal{S H}(\Omega) \subseteq$ $\mathcal{S H}(\Omega)$ and $\mathcal{P} \mathcal{H}(\Omega) \subseteq \mathcal{H}(\Omega)$. If $n=1$, then the inclusions are equalities, and if $n>1$, they are proper. We now define what it means for a function to be plurisubharmonic and pluriharmonic on the boundary of a bounded hyperconvex product domain.

Definition 3.1. Let $D \subseteq \mathbb{C}^{n}$ be a bounded hyperconvex product domain as in (2.1). An upper semicontinuous function $u: \partial D \rightarrow \mathbb{R} \cup\{-\infty\}$ is plurisubharmonic if $u$ is plurisubharmonic on every $\widehat{\Lambda}^{J}$, i.e., for all $k \in$ $\{1, \ldots, s-1\}, 1 \leq j_{1}<\cdots<j_{k} \leq s$ and $\left(z_{j_{1}}, \ldots, z_{j_{k}}\right) \in \partial D_{j_{1}} \times \cdots \times \partial D_{j_{k}}$, the function defined by

$$
\left(z_{1}, \ldots, \widehat{z}_{j_{1}}, \ldots, \widehat{z}_{j_{k}}, \ldots, z_{s}\right) \mapsto u\left(z_{1}, \ldots, z_{j_{1}}, \ldots, z_{j_{k}}, \ldots, z_{s}\right)
$$

is plurisubharmonic on the open set $\widehat{\Lambda}^{J} \subseteq \mathbb{C}^{n-n_{J}}$, where $n_{J}=n_{j_{1}}+\cdots+n_{j_{k}}$. The identically $-\infty$ function is by fiat not considered as plurisubharmonic. In a similar manner a continuous function $u: \partial D \rightarrow \mathbb{R}$ is pluriharmonic if it is pluriharmonic on each $\widehat{\Lambda}^{J}$.

EXAmple 3.2. Let $P$ be the unit polydisc in $\mathbb{C}^{n}, n \geq 2$, and consider the function $f: \partial P \rightarrow \mathbb{R}$ defined by

$$
f(z)=f\left(z_{1}, \ldots, z_{n}\right)= \begin{cases}0 & \text { if }\left|z_{j}\right|=1 \text { for every } j \\ 1 & \text { otherwise. }\end{cases}
$$

Then $f$ is harmonic on every analytic disc embedded in $\partial P$, but it is not pluriharmonic on $\partial P$ in the sense of Definition 3.1 since it is not continuous on $\partial P$. Moreover, there does not exist a pluriharmonic function $h$ such that $\left.h\right|_{\partial D}=f$.

THEOREM 3.3. Let $D_{j}$ be a bounded hyperconvex domain in $\mathbb{C}^{n_{j}}, j=$ $1, \ldots, s, s \geq 3$, set $D=D_{1} \times \cdots \times D_{s}, n=n_{1}+\cdots+n_{s}$, and let $f: \partial D \rightarrow \mathbb{R}$ be a continuous function. The following are then equivalent: 
(1) there exists a function $u$ which is pluriharmonic on D, continuous on $\bar{D}$ and $\left.u\right|_{\partial D}=f$,

(2) $f$ is pluriharmonic on $\partial D$ in the sense of Definition 3.1,

(3) the Perron-Bremermann envelope $P B_{f}$ is pluriharmonic on $D$, i.e.,

$$
P B_{-f}=-P B_{f},
$$

(4) for every $z_{0} \in \partial D$ and every Jensen measure $\mu$ with barycenter $z_{0}$,

$$
f\left(z_{0}\right)=\int_{\partial D} f d \mu
$$

Proof. $(1) \Rightarrow(2)$ : Let $k \in\{1, \ldots, s-1\}, J$ be an increasing multi-index, $1 \leq j_{1}<\cdots<j_{k} \leq s$, and let $\left(\xi_{j_{1}}, \ldots, \xi_{j_{k}}\right) \in \partial D_{j_{1}} \times \cdots \times \partial D_{j_{k}}$. Define the function $f_{\xi_{1}, \ldots, \xi_{k}}: \widehat{\Lambda}^{J} \rightarrow \mathbb{R}$ by

$$
f_{\xi_{1}, \ldots, \xi_{k}}\left(z_{1}, \ldots, \widehat{\xi}_{j_{1}}, \ldots, \widehat{\xi}_{j_{k}}, \ldots, z_{s}\right)=f\left(z_{1}, \ldots, \xi_{j_{1}}, \ldots, \xi_{j_{k}}, \ldots, z_{s}\right) .
$$

Take $z_{0} \in \widehat{\Lambda}^{J}$ and let $\widetilde{z}_{0}=\left(z_{1}, \ldots, \xi_{j_{1}}, \ldots, \xi_{j_{k}}, \ldots, z_{s}\right) \in \Lambda^{J}$ and in the same way take $X \in \mathbb{C}^{n-n_{J}}$ and let $\tilde{X}=\left(X_{1}, \ldots, X_{j_{l}}, \ldots, X_{n}\right)$, where $X_{j_{l}}=0$ for $l=1, \ldots, n_{J}$. Choose $r>0$ such that $B_{r}=\left\{\widetilde{z}_{0}+\zeta r \widetilde{X}: \zeta \in \mathbb{C},|\zeta|<1\right\}$ $\subseteq \Lambda^{J}$. Let $D_{j_{l}} \ni \xi_{j_{l}}^{m} \rightarrow \xi_{j_{l}}$ as $m \rightarrow \infty$, for $1 \leq l \leq k$, and let $\widetilde{z}_{0}^{m}=$ $\left(z_{1}, \ldots, \xi_{j_{1}}^{m}, \ldots, \xi_{j_{k}}^{m}, \ldots, z_{s}\right) \in D$. For each $m$ define $u_{m}(\zeta)=u\left(\widetilde{z}_{0}^{m}+\zeta r \tilde{X}\right)$. Then $u_{m}$ is harmonic on the unit disc in $\mathbb{C}$, by the assumption that $u \in$ $\mathcal{P H}(D) \cap C(\bar{D})$. Moreover, $u_{m}$ converges uniformly to $f_{\xi_{1}, \ldots, \xi_{k}}$ as $m \rightarrow \infty$. Thus, $f_{\xi_{1}, \ldots, \xi_{k}}$ is harmonic on $B_{r}$ and therefore pluriharmonic on $\Lambda^{J}$.

$(2) \Rightarrow(1):$ Assume that $f: \partial D \rightarrow \mathbb{R}$ is a continuous function and set

$$
u\left(z_{1}, \ldots, z_{s}\right)=\int_{\partial D^{+}} f\left(t_{1}, \ldots, t_{s}\right) d \omega_{z_{1}}\left(t_{1}\right) \cdots d \omega_{z_{s}}\left(t_{s}\right),
$$

where $\omega_{z_{j}}$ is the harmonic measure relative to $D_{j}$ and $z_{j}$. Then $u$ is $s$ harmonic on $D$, continuous on $\bar{D}$ and $\left.u\right|_{\partial D}=f$. We now show that $u$ is pluriharmonic on $D$. Let $z_{0}=\left(z_{1}, \ldots, z_{s}\right) \in D, X=\left(X_{1}, \ldots, X_{s}\right) \in \mathbb{C}^{n}$ $\left(X_{j} \in \mathbb{C}^{n_{j}}\right)$ be such that $X_{s}=0$ and choose $r>0$ such that $\left\{z_{0}+\zeta X\right.$ : $\zeta \in \mathbb{C},|\zeta|<r\} \subseteq D$. Let $B_{r}=B(0, r) \subset \mathbb{C}$ be the Euclidean ball with centre 0 and radius $r$. For any $w_{1} \in D_{1}, \ldots, w_{s-1} \in D_{s-1}, w_{s} \in \partial D_{s}, w^{\prime}=$ $\left(w_{1}, \ldots, w_{s-1}\right), X^{\prime}=\left(X_{1}, \ldots, X_{s-1}\right), \zeta \in B_{r}, t^{\prime}=\left(t_{1}, \ldots, t_{s-1}\right)$, where $t_{j} \in D_{j}, 1 \leq j \leq s-1$ define

$$
d \omega_{w^{\prime}+\zeta X^{\prime}}^{\prime}\left(t^{\prime}\right)=d \omega_{w_{1}+\zeta X_{1}}\left(t_{1}\right) \cdots d \omega_{w_{s-1}+\zeta X_{s-1}}\left(t_{s-1}\right) .
$$

The assumption that $f$ is pluriharmonic in the sense of Definition 3.1 implies in particular that $f$ is pluriharmonic on $D_{1} \times \cdots \times D_{s-1} \times\left\{w_{s}\right\}$, hence

$$
\frac{1}{\pi r^{2}} \int_{B_{r}} f\left(w^{\prime}+\zeta X^{\prime}, w_{s}\right) d \lambda(\zeta)=f\left(w^{\prime}, w_{s}\right)=\int_{\partial D^{+}} f d \omega_{w_{1}} \cdots d \omega_{w_{s}}
$$


and

$$
f\left(w^{\prime}+\zeta X^{\prime}, w_{s}\right)=\int_{\partial D_{1} \times \cdots \times \partial D_{s-1}} f\left(t^{\prime}, w_{s}\right) d \omega_{w^{\prime}+\zeta X^{\prime}}^{\prime}
$$

Therefore,

$$
\begin{aligned}
\frac{1}{\pi r^{2}} \int_{B_{r}} u\left(z_{0}+\zeta X\right) d \lambda(\zeta)=\frac{1}{\pi r^{2}} \int_{B_{r}} \int_{\partial D^{+}} f\left(t^{\prime}, t_{s}\right) d \omega_{z^{\prime}+\zeta X^{\prime}}^{\prime}\left(t^{\prime}\right) d \omega_{z_{s}}\left(t_{s}\right) d \lambda(\zeta) \\
=\int_{\partial D_{s}} \frac{1}{\pi r^{2}} \int_{B_{r} \partial D_{1} \times \cdots \times \partial D_{s-1}} f\left(t^{\prime}, t_{s}\right) d \omega_{z^{\prime}+\zeta X^{\prime}}^{\prime}\left(t^{\prime}\right) d \lambda(\zeta) d \omega_{z_{s}}\left(t_{s}\right) \\
=\int_{\partial D_{s}} \frac{1}{\pi r^{2}} \int_{B_{r}} f\left(z^{\prime}+\zeta X^{\prime}, t_{s}\right) d \lambda(\zeta) d \omega_{z_{s}}\left(t_{s}\right)=\int_{\partial D_{s}} f\left(z^{\prime}, t_{s}\right) d \omega_{z_{s}}\left(t_{s}\right) \\
=\int_{\partial D_{1} \times \cdots \times \partial D_{s}} f\left(t_{1}, \ldots, t_{s}\right) d \omega_{z_{1}}\left(t_{1}\right) \cdots d \omega_{z_{s}}\left(t_{s}\right)=u\left(z_{0}\right)
\end{aligned}
$$

which proves that $u$ is pluriharmonic on $D_{1} \times \cdots \times D_{s-1} \times\left\{z_{s}\right\}$ for all $z_{s} \in D_{s}$. By repeating the same argument for $X \in \mathbb{C}^{n}$ such that $X_{k}=0$, $1 \leq k \leq s-1$, we reach the conclusion that for each $k$ fixed, $1 \leq k \leq s$, the function $u$ is pluriharmonic on

$$
D_{1} \times \cdots \times\left\{z_{k}\right\} \times \cdots \times D_{s} \subseteq D,
$$

for all $z_{k} \in D_{k}$. This means that $u$ is pluriharmonic on $D$ since

$$
\begin{aligned}
& \mathcal{L} u\left(z_{1}, \ldots, z_{s}\right)\left(X_{1}, \ldots, X_{s}\right) \\
& =\frac{1}{s-2} \sum_{j=1}^{s} \mathcal{L}\left(u \circ \varrho_{j}\right)\left(z_{1}, \ldots, z_{j-1}, z_{j+1}, \ldots, z_{s}\right)\left(X_{1}, \ldots, X_{j-1}, X_{j+1}, \ldots, X_{s}\right) \\
& \quad-\frac{1}{s-2} \sum_{k=1}^{n_{1}+\cdots+n_{s}} \frac{\partial^{2} u}{\partial z_{k} \partial \bar{z}_{k}}\left(z_{1}, \ldots, z_{s}\right)\left|X_{k}\right|^{2}=0,
\end{aligned}
$$

where $\left(z_{1}, \ldots, z_{s}\right) \in D, X=\left(X_{1}, \ldots, X_{s}\right) \in \mathbb{C}^{n}, X_{j} \in \mathbb{C}^{n_{j}}, \varrho_{j}\left(z_{1}, \ldots, \widehat{z}_{j}, \ldots, z_{s}\right)$ $=\left(z_{1}, \ldots, z_{j}, \ldots, z_{s}\right), \mathcal{L} u$ is the Levi form of $u, X_{j} \in \mathbb{C}^{n_{j}}$ and $s \geq 3$.

$(2) \Rightarrow(3)$ : Assume that $f: \partial D \rightarrow \mathbb{R}$ is pluriharmonic. The proof of (2) $\Rightarrow(1)$ shows that the function $u$ defined by

$$
u\left(z_{1}, \ldots, z_{s}\right)=\int_{\partial D^{+}} f\left(t_{1}, \ldots, t_{s}\right) d \omega_{z_{1}}\left(t_{1}\right) \cdots d \omega_{z_{s}}\left(t_{s}\right)
$$

is pluriharmonic on $D$, continuous on $\bar{D}$ and $\left.u\right|_{\partial D}=f$. Hence $\left(d d^{c} u\right)^{n}=0$ and therefore $u=P B_{f}$ (see e.g. [7]). By the same arguments the function $v$ defined by

$$
v\left(z_{1}, \ldots, z_{s}\right)=\int_{\partial D^{+}}\left(-f\left(t_{1}, \ldots, t_{s}\right)\right) d \omega_{z_{1}}\left(t_{1}\right) \cdots d \omega_{z_{s}}\left(t_{s}\right)
$$


is pluriharmonic on $D$, continuous on $\bar{D}$ and $\left.v\right|_{\partial D}=-f$. Thus $v=P B_{-f}$ (see e.g. [7]), which implies that $P B_{-f}=-P B_{f}$ on $D$, by the construction of $u$ and $v$.

$(3) \Rightarrow(4)$ : This is a direct consequence of Theorem 2.4 .

$(4) \Rightarrow(2)$ : Fix $k \in\{1, \ldots, s-1\}$ and let $J, 1 \leq j_{1}<\cdots<j_{k} \leq s$, be an increasing multi-index. Take $z_{0} \in \widehat{\Lambda}^{J}$ and let $\widetilde{z}_{0}=\left(z_{1}, \ldots, \xi_{j_{1}}, \ldots, \xi_{j_{k}}, \ldots, z_{s}\right)$ $\in \Lambda^{J}$, where $\left(\xi_{j_{1}}, \ldots, \xi_{j_{k}}\right) \in \partial D_{j_{1}} \times \cdots \times \partial D_{j_{k}}$. In the same way take $X \in \mathbb{C}^{n-n_{J}}$ and let $\widetilde{X}=\left(X_{1}, \ldots, X_{j_{l}}, \ldots, X_{n}\right)$, where $X_{j_{l}}=0$ for $l=$ $1, \ldots, n_{J}$. Choose $r>0$ such that $A=\left\{\widetilde{z}_{0}+\zeta \widetilde{X}: \zeta \in \mathbb{C},|\zeta|<r\right\} \subseteq \Lambda^{J}$. If $\mu_{0}=(2 \pi r)^{-1} d \lambda$, where $\lambda$ is the Lebesgue measure on $A$, then $\mu_{0} \in \mathcal{J}_{\widetilde{z}_{0}}$ and supp $\mu_{0} \subseteq A$. Thus,

$$
f\left(\widetilde{z}_{0}\right)=\int_{\partial D} f d \mu_{0}=\int_{A} f d \mu_{0}
$$

by assumption, and therefore $f$ is harmonic in $A$, which implies that it is harmonic in $\Lambda^{J}$.

Example 3.4 below shows that for $n=2$ the implication $(2) \Rightarrow(1)$ in Theorem 3.3 is, in general, not true. The construction of the function $u$ in this example is due to Poletsky ([25]).

Example 3.4. Let $\mathbb{D}^{2}=\left\{(z, w) \in \mathbb{C}^{2}:|z|<1,|w|<1\right\}$ be the unit polydisc in $\mathbb{C}^{2}$ and let $f: \partial \mathbb{D}^{2} \rightarrow \mathbb{R}$ be defined by

$$
f(\zeta, \xi)=\operatorname{Re}(\zeta \bar{\xi})
$$

Then $f$ is pluriharmonic on $\partial \mathbb{D}^{2}$ in the sense of Definition 3.1; we will prove that condition (1) in Theorem 3.3 is not true for $f$. Let $u$ be a function defined on $\mathbb{D}^{2}$ by

$$
u(z, w)=\frac{\operatorname{Re}(z \bar{w})\left(2-|z|^{2}-|w|^{2}\right)}{1-|z w|^{2}}-\frac{\left(1-|z|^{2}\right)\left(1-|w|^{2}\right)}{1-|z w|^{2}} .
$$

Then $u \in C^{\infty}\left(\mathbb{D}^{2}\right) \cap C\left(\overline{\mathbb{D}}^{2}\right)$ and $\lim _{(z, w) \rightarrow(\zeta, \xi)} u(z, w)=\operatorname{Re}(\zeta \bar{\xi})$ for every $(\zeta, \xi) \in \partial \mathbb{D}^{2}$. We have

$$
\begin{aligned}
& u_{z \bar{z}}(z, w)=\frac{\partial^{2} u}{\partial z \partial \bar{z}}(z, w)=\frac{\left(1-|w|^{2}\right)^{2}|1-z \bar{w}|^{2}}{\left(1-|z|^{2}|w|^{2}\right)^{3}} \\
& u_{z \bar{w}}(z, w)=\frac{\partial^{2} u}{\partial z \partial \bar{w}}(z, w)=\frac{\left(1-|z|^{2}\right)\left(1-|w|^{2}\right)(1-\bar{z} w)^{2}}{\left(1-|z|^{2}|w|^{2}\right)^{3}} \\
& u_{\bar{z} w}(z, w)=\frac{\partial^{2} u}{\partial \bar{z} \partial w}(z, w)=\frac{\left(1-|z|^{2}\right)\left(1-|w|^{2}\right)(1-z \bar{w})^{2}}{\left(1-|z|^{2}|w|^{2}\right)^{3}} \\
& u_{w \bar{w}}(z, w)=\frac{\partial^{2} u}{\partial w \partial \bar{w}}(z, w)=\frac{\left(1-|z|^{2}\right)^{2}|1-\bar{z} w|^{2}}{\left(1-|z|^{2}|w|^{2}\right)^{3}}
\end{aligned}
$$


Since $u_{z \bar{z}} \geq 0, u_{w \bar{w}} \geq 0$ and

$$
\operatorname{det}\left(\begin{array}{cc}
u_{z \bar{z}}(z, w) & u_{z \bar{w}}(z, w) \\
u_{\bar{z} w}(z, w) & u_{w \bar{w}}(z, w)
\end{array}\right)=0,
$$

it follows that $u$ is a maximal plurisubharmonic function on $\mathbb{D}^{2}$ and $u=$ $P B_{f}$ (see e.g. [7]). We will next obtain an explicit formula for $P B_{-f}$. Let $F(z, w)=(-z, w)$. Then $-\operatorname{Re}(z \bar{w})=\operatorname{Re}(z \bar{w}) \circ F$ and $P B_{-f}=P B_{f} \circ F$. Thus,

$$
P B_{-f}(z, w)=\frac{-\operatorname{Re}(z \bar{w})\left(2-|z|^{2}-|w|^{2}\right)}{1-|z w|^{2}}-\frac{\left(1-|z|^{2}\right)\left(1-|w|^{2}\right)}{1-|z w|^{2}}
$$

and we see that $P B_{f}+P B_{-f} \neq 0$, hence $P B_{f}$ is not pluriharmonic on $\mathbb{D}^{2}$ and therefore condition (1) in Theorem 3.3 is not true for $f$.

DEFINITION 3.5. A compliant function is a continuous function $f: \partial \Omega \rightarrow \mathbb{R}$ with the following two properties:

- $\lim _{z \rightarrow \xi, z \in \Omega}\left(P B_{f}+P B_{-f}\right)(z)=0$ for every $\xi \in \partial \Omega$,

- $\int_{\Omega}\left(d d^{c}\left(P B_{f}+P B_{-f}\right)\right)^{n}<\infty$,

where $\left(d d^{c} \cdot\right)^{n}$ is the complex Monge-Ampère operator. Let $\mathcal{C P}(\partial \Omega)$ denote the class of compliant functions on $\partial \Omega$.

Let $u$ be a holomorphic function defined in a neighbourhood of $\bar{\Omega}$ and let $f=\operatorname{Re}(u)$ on $\partial \Omega$. Then $f$ is an elementary example of a compliant function. For further information and examples see [2]. Corollary 3.6 below follows immediately from Theorem 3.3.

Corollary 3.6. Let $D_{j}$ be a bounded hyperconvex domain in $\mathbb{C}^{n_{j}}, j=$ $1, \ldots, s, s \geq 3$, and set $D=D_{1} \times \cdots \times D_{s}$. Then $\mathcal{C P}(\partial D)=\mathcal{P} \mathcal{H}(\partial D)$.

Example 3.7 below shows that there exists a compliant function $f$ for which $P B_{f}$ is not pluriharmonic. Note that when $n \geq 3$ this is not possible (Theorem 3.3).

EXAmple 3.7. Let $f$ be defined as in Example 3.4. Condition (2) in Theorem A is satisfied for $f$ and therefore so is condition (4). From Theorem 2.4 it follows that

$$
\lim _{z \rightarrow \xi, z \in \Omega}\left(P B_{f}+P B_{-f}\right)(z)=0
$$

for every $\xi \in \partial \Omega$. By some straightforward calculations we get

$$
\left(d d^{c}\left(P B_{f}+P B_{-f}\right)\right)^{2}=128 \frac{\left(1-|z|^{2}\right)^{2}\left(1-|w|^{2}\right)^{2}}{\left(1-|z|^{2}|w|^{2}\right)^{4}} d \lambda_{2}
$$


and

$$
\int_{\mathbb{D}^{2}}\left(d d^{c}\left(P B_{f}+P B_{-f}\right)\right)^{2}=128 \int_{\mathbb{D}^{2}} \frac{\left(1-|z|^{2}\right)^{2}\left(1-|w|^{2}\right)^{2}}{\left(1-|z|^{2}|w|^{2}\right)^{4}} d \lambda_{2}(z, w)=\frac{64 \pi^{2}}{3}
$$

where $\lambda_{2}$ is the Lebesgue measure on $\mathbb{C}^{2}$. Thus, $f$ is compliant.

We end this section by proving a sufficient condition for a continuous function defined on $\partial \mathbb{D}^{2}$ to be compliant.

Proposition 3.8. If $f: \partial \mathbb{D}^{2} \rightarrow \mathbb{R}$ is a pluriharmonic function in the sense of Definition 3.1 which satisfies

$$
\sum_{k_{1}, k_{2}=0}^{\infty} \sqrt{k_{1} k_{2}}\left|a_{k_{1}, k_{2}}\right|<\infty
$$

then $f$ is compliant. Here

$$
a_{k_{1}, k_{2}}=\int_{\partial \mathbb{D} \times \partial \mathbb{D}} w_{1}^{k_{1}} \bar{w}_{2}^{k_{2}} f\left(w_{1}, w_{2}\right) d \sigma\left(w_{1}\right) d \sigma\left(w_{2}\right),
$$

where $d \sigma$ is the normalized Lebesgue measure on $\partial \mathbb{D}$.

Proof. For any integers $k, l \geq 1$ let $f_{k, l}=\operatorname{Re}\left(\zeta^{k} \bar{\xi}^{l}\right)$ and $g_{k, l}=\operatorname{Im}\left(\zeta^{k} \bar{\xi}^{l}\right)$. Then Example 3.7 shows that

$$
\begin{aligned}
\int_{\mathbb{D}^{2}}\left(d d^{c}\left(P B_{f_{k, l}}+P B_{-f_{k, l}}\right)\right)^{2} & =\int_{\mathbb{D}^{2}}\left(d d^{c}\left(P B_{g_{k, l}}+P B_{-g_{k, l}}\right)\right)^{2} \\
& =k l \int_{\mathbb{D}^{2}}\left(d d^{c}\left(P B_{f_{1,1}}+P B_{-f_{1,1}}\right)\right)^{2}=\frac{64 \pi^{2} k l}{3},
\end{aligned}
$$

and therefore $f_{k, l}$ and $g_{k, l}$ are compliant. Let

$$
u\left(z_{1}, z_{2}\right)=\int_{\partial \mathbb{D} \times \partial \mathbb{D}} \frac{\left(1-\left|z_{1}\right|^{2}\right)\left(1-\left|z_{2}\right|^{2}\right)}{\left|w_{1}-z_{1}\right|^{2}\left|w_{2}-z_{2}\right|^{2}} f\left(w_{1}, w_{2}\right) d \sigma\left(w_{1}\right) d \sigma\left(w_{2}\right),
$$

Then $u$ is 2-harmonic on $\mathbb{D}$, continuous on $\overline{\mathbb{D}}$ and $\left.u\right|_{\partial \mathbb{D}}=f$. Note that $u$ is, in general, not pluriharmonic. There exists a holomorphic function $U$ defined on $\mathbb{D}^{2}$ such that

$$
u\left(z_{1}, z_{2}\right)=\operatorname{Re}(U)+\sum_{k_{1}, k_{2}=0}^{\infty} a_{k_{1}, k_{2}} \bar{z}_{1}^{k_{1}} z_{2}^{k_{2}}+\sum_{k_{1}, k_{2}=0}^{\infty} \bar{a}_{k_{1}, k_{2}} z_{1}^{k_{1}} \bar{z}_{2}^{k_{2}}
$$

(see e.g. [26]), hence

$$
f\left(z_{1}, z_{2}\right)=\operatorname{Re}(U)+\sum_{k_{1}, k_{2}=0}^{\infty} b_{k_{1}, k_{2}} \operatorname{Re}\left(z_{1}^{k_{1}} \bar{z}_{2}^{k_{2}}\right)+\sum_{k_{1}, k_{2}=0}^{\infty} c_{k_{1}, k_{2}} \operatorname{Im}\left(z_{1}^{k_{1}} \bar{z}_{2}^{k_{2}}\right),
$$


where $b_{k_{1}, k_{2}}=\operatorname{Re}\left(a_{k_{1}, k_{2}}\right)$ and $c_{k_{1}, k_{2}}=-\operatorname{Im}\left(a_{k_{1}, k_{2}}\right)$. Now it follows from [2] that

$$
\begin{aligned}
& \left(\int_{\mathbb{D}^{2}}\left(d d^{c}\left(P B_{f}+P B_{-f}\right)\right)^{2}\right)^{1 / 2} \\
& \leq \sum_{k_{1}, k_{2}=0}^{\infty}\left|b_{k_{1}, k_{2}}\right|\left(\int_{\mathbb{D}^{2}}\left(d d^{c} v\left(f_{k_{1}, k_{2}}\right)\right)^{2}\right)^{1 / 2}+\sum_{k_{1}, k_{2}=0}^{\infty}\left|c_{k_{1}, k_{2}}\right|\left(\int_{\mathbb{D}^{2}}\left(d d^{c} v\left(g_{k_{1}, k_{2}}\right)\right)^{2}\right)^{1 / 2} \\
& \leq \frac{8 \pi}{\sqrt{3}} \sum_{k_{1}, k_{2}=0}^{\infty} \sqrt{k_{1} k_{2}}\left(\left|b_{k_{1}, k_{2}}\right|+\left|c_{k_{1}, k_{2}}\right|\right) \leq \frac{16 \pi}{\sqrt{3}} \sum_{k_{1}, k_{2}=0}^{\infty} \sqrt{k_{1} k_{2}}\left|a_{k_{1}, k_{2}}\right|<\infty
\end{aligned}
$$

where $v\left(f_{k_{1}, k_{2}}\right)=P B_{f_{k_{1}, k_{2}}}+P B_{-f_{k_{1}, k_{2}}}$ and $v\left(g_{k_{1}, k_{2}}\right)=P B_{g_{k_{1}, k_{2}}}+P B_{-g_{k_{1}, k_{2}}}$. Thus, $f$ is compliant.

4. Plurisubharmonic boundary values and analytic discs. In this section we will prove a characterization of plurisubharmonic functions defined on the boundary in terms of analytic discs. Let $\Omega \subseteq \mathbb{C}^{n}$ be a bounded domain. By an analytic disc embedded in $\partial \Omega$ we mean an injective, holomorphic function $d: \mathbb{D} \rightarrow \partial \Omega$, where $\mathbb{D}$ is the unit disc in $\mathbb{C}$.

Proposition 4.1. Let $D=D_{1} \times \cdots \times D_{n} \subset \mathbb{C}^{n}, n \geq 2$, be a bounded hyperconvex domain, where $D_{j} \subseteq \mathbb{C}, 1 \leq j \leq n$. Then for every analytic disc $d: \mathbb{D} \rightarrow \partial D$ there exist $k \in\{1, \ldots, n-1\}$ and an increasing multi-index $J$, $1 \leq j_{1}<\cdots<j_{k} \leq n$, such that

$$
d(\mathbb{D}) \subseteq D_{1} \times \cdots \times \partial D_{j_{1}} \times \cdots \times \partial D_{j_{k}} \times \cdots \times D_{n}=\Lambda^{J} .
$$

Proof. Let $d: \mathbb{D} \rightarrow \partial D$ be an analytic disc. Then $d(\mathbb{D})$ is a connected manifold of real dimension 2 so $d(\mathbb{D}) \nsubseteq \partial D^{+}$. Assume that $d(\mathbb{D}) \cap \partial D^{+} \neq \emptyset$. Then there exist $w \in d(\mathbb{D}) \cap \partial D^{+}$and $z_{j} \in d(\mathbb{D}) \cap\left(\partial D \backslash \partial D^{+}\right)$such that $\Pi_{j}\left(z_{j}\right) \in D_{j}$, where $\Pi_{j}$ is the standard projection on the $j$ th coordinate. Therefore there exist $\zeta_{1}, \zeta_{2} \in \mathbb{D}$ such that $\Pi_{j} \circ d\left(\zeta_{1}\right) \in \partial D_{j}$ and $\Pi_{j} \circ d\left(\zeta_{2}\right) \in$ $D_{j}$, which is impossible since $\Pi_{j} \circ d$ is an open map. Thus $d(\mathbb{D}) \cap \partial D^{+}=\emptyset$. Now since $d(\mathbb{D})$ is connected there exist $k \in\{1, \ldots, n-1\}$ and an increasing multi-index $J, 1 \leq j_{1}<\cdots<j_{k} \leq n$, such that $d(\mathbb{D}) \subseteq \Lambda^{J}$.

Theorem 4.2 will be a prominent tool in Section 5 .

Theorem 4.2. Let $D=D_{1} \times \cdots \times D_{n} \subset \mathbb{C}^{n}, n \geq 2$, be a bounded hyperconvex domain, where $D_{j} \subseteq \mathbb{C}, 1 \leq j \leq n$, and let $f: \partial D \rightarrow \mathbb{R}$ be a continuous function. The following are then equivalent:

(1) $f$ is plurisubharmonic in the sense of Definition 3.1,

(2) $f$ is subharmonic on every analytic disc d embedded in $\partial D$, i.e., $f \circ d$ is subharmonic on $\mathbb{D} \subseteq \mathbb{C}$,

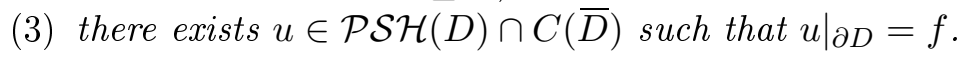


Proof. $(2) \Rightarrow(1)$ : Let $k \in\{1, \ldots, n-1\}, J$ an increasing multi-index, $1 \leq j_{1}<\cdots<j_{k} \leq n$, and $\left(\xi_{j_{1}}, \ldots, \xi_{j_{k}}\right) \in \partial D_{j_{1}} \times \cdots \times \partial D_{j_{k}}$. Define $f_{\xi_{1}, \ldots, \xi_{k}}: \widehat{\Lambda}^{J} \rightarrow \mathbb{R}$ by

$$
f_{\xi_{1}, \ldots, \xi_{k}}\left(z_{1}, \ldots, \widehat{\xi}_{j_{1}}, \ldots, \widehat{\xi}_{j_{k}}, \ldots, z_{n}\right)=f\left(z_{1}, \ldots, \xi_{j_{1}}, \ldots, \xi_{j_{k}}, \ldots, z_{n}\right) .
$$

Take $z_{0} \in \widehat{\Lambda}^{J}$ and let $\widetilde{z}_{0}=\left(z_{1}, \ldots, \xi_{j_{1}}, \ldots, \xi_{j_{k}}, \ldots, z_{s}\right) \in \Lambda^{J}$; moreover, take $X \in \mathbb{C}^{n-k}$ and let $\widetilde{X}=\left(X_{1}, \ldots, X_{j_{l}}, \ldots, X_{n}\right)$, where $X_{j_{l}}=0$ for $l=1, \ldots, k$. Choose $r>0$ such that $\left\{\widetilde{z}_{0}+\zeta r \widetilde{X}: \zeta \in \mathbb{C},|\zeta|<1\right\} \subseteq \Lambda^{J}$. Define $d: \mathbb{D} \rightarrow \Lambda^{J}$ by $d(\zeta)=\widetilde{z}_{0}+\zeta r \widetilde{X}$. Then $d$ is an analytic disc embedded in $\Lambda^{J}$. Thus $f \circ d$ is subharmonic on $\mathbb{D}$ by assumption, hence $f_{\xi_{1}, \ldots, \xi_{k}}$ is plurisubharmonic on $\widehat{\Lambda}^{J}$.

$(1) \Rightarrow(2)$ : Let $d$ be an analytic disc embedded in $\partial D$. Proposition 4.1 shows that there exists a $k \in\{1, \ldots, n-1\}$ and an increasing multi-index $J, 1 \leq j_{1}<\cdots<j_{k} \leq n$, such that $d(\mathbb{D}) \subseteq \Lambda^{J}$. By definition, $f$ is plurisubharmonic on $\widehat{\Lambda}^{J}$, which implies that $f \circ d$ is subharmonic on $\mathbb{D}$.

$(3) \Rightarrow(1)$ : See the proof of Theorem 3.3.

$(1) \Rightarrow(3)$ : Just take $u=P B_{f}$. Observe also that $\mathcal{J}_{z_{0}}=\left\{\delta_{z_{0}}\right\}$ for all $z_{0} \in \partial D^{+}$, so by (1) we get, for all $z \in \partial D$,

$$
f(z)=\inf \left\{\int_{\partial D} f d \mu_{z}: \mu_{z} \in \mathcal{J}_{z_{0}}\right\} .
$$

Therefore Theorem 3.5 in [30] implies that $u \in \mathcal{P S \mathcal { H }}(D) \cap C(\bar{D})$ and $\left.u\right|_{\partial D}=f$.

REMARK. In [8] Błocki proved the equivalence of (2) and (3) in the case when $D$ is the unit polydisc in $\mathbb{C}^{n}$. See also [28] and for the case when $n=2$, Example 3.6 in [30].

The following is well-known (cf. e.g. the proof of Corollary 2.10 in [9]).

LEMMA 4.3. Let $\Omega$ be a bounded domain in $\mathbb{C}^{n}$ with $C^{1}$-boundary and $h: \mathbb{D} \rightarrow \bar{\Omega}$ a holomorphic function. If $u \in \mathcal{P} \mathcal{S H}(\Omega) \cap C(\bar{\Omega})$, then $u \circ h$ is subharmonic on $\mathbb{D}$.

Proof. By [18] there exists a sequence $\left[u_{j}\right]$ of smooth plurisubharmonic functions defined on a neighbourhood of $\bar{\Omega}$ which converges uniformly to $u$ on $\bar{\Omega}$ as $j \rightarrow \infty$. Hence, $u_{j} \circ h$ subharmonic on $\mathbb{D}$ and therefore $u \circ h$ is subharmonic on $\mathbb{D}$, since $u_{j} \circ h$ converges uniformly on $\bar{\Omega}$ to $u \circ h$ as $j \rightarrow \infty$.

REMARK. It is not known to the authors if Lemma 4.3 is valid for more general domains.

THEOREM 4.4. Let $D=D_{1} \times \cdots \times D_{s}$ be a bounded hyperconvex product domain, where each $D_{j}$ is a bounded hyperconvex domain in $\mathbb{C}^{n_{j}}$ with $C^{1}$ boundary, $1 \leq j \leq s$ and $s \geq 2$. For every continuous function $f: \partial D \rightarrow \mathbb{R}$ the following assertions are equivalent: 
(1) $f$ is plurisubharmonic in the sense of Definition 3.1,

(2) $f$ is subharmonic on every analytic disc d embedded in $\partial D$, i.e., $f \circ d$ is subharmonic on $\mathbb{D}$.

Proof. The implication $(2) \Rightarrow(1)$ follows as in the proof of Theorem 4.2. For the converse let $d: \mathbb{D} \rightarrow \partial D$ be an analytic disc. Assume that $d(\mathbb{D}) \cap$ $\partial D^{+} \neq \emptyset$ and $d(\mathbb{D}) \cap\left(\partial D \backslash \partial D^{+}\right) \neq \emptyset$. Then there exist $j$ and $z_{1} \in \partial D_{j}$, $z_{2} \in D_{j}$ such that $z_{1}, z_{2} \in d(\mathbb{D})$. Hence, $d(\mathbb{D}) \cap \partial D_{j} \neq \emptyset$ and $d(\mathbb{D}) \cap D_{j} \neq \emptyset$, which contradicts Corollary 2.10 in [9], since $D_{j}$ is a hyperconvex domain with $C^{1}$-boundary. Thus either $d(\mathbb{D}) \subseteq \partial D^{+}$or $d(\mathbb{D}) \subset \partial D \backslash \partial D^{+}$. If $d(\mathbb{D}) \subseteq$ $\partial D \backslash \partial D^{+}$, then there exists a $k \in\{1, \ldots, s-1\}$ and an increasing multiindex $J, 1 \leq j_{1}<\cdots<j_{k} \leq s$, such that $d(\mathbb{D}) \subseteq \Lambda^{J}$, since $d(\mathbb{D})$ is a connected manifold of real dimension 2. Therefore $f \circ d$ is subharmonic by (1). Similarly, if $d(\mathbb{D}) \subseteq \partial D^{+}$, then there exists $1 \leq j \leq s$ such that $d(\mathbb{D}) \subseteq \partial D_{j}$. This implies that $f \circ d$ is subharmonic by Lemma 4.3 .

\section{Pluriharmonic boundary values on the symmetrized polydisc.}

In this section we prove Theorem A for the case when $\Omega$ is the symmetrized polydisc $\mathbb{G}_{n}$. Let $\pi_{n}=\left(\pi_{n, 1}, \ldots, \pi_{n, n}\right): \mathbb{C}^{n} \rightarrow \mathbb{C}^{n}, n \geq 1$, be defined as follows:

$$
\pi_{n, k}\left(z_{1}, \ldots, z_{n}\right)=\sum_{1 \leq j_{1}<\cdots<j_{k} \leq n} z_{j_{1}} \cdots z_{j_{k}}
$$

for $1 \leq k \leq n$. Then $\pi_{n}$ is a proper holomorphic mapping with multiplicity $n$ !, and so also is $\left.\pi_{n}\right|_{\mathbb{D}^{n}}: \mathbb{D}^{n} \rightarrow \pi_{n}\left(\mathbb{D}^{n}\right)$. Moreover, $\pi_{n}\left(\partial \mathbb{D}^{n}\right)=\partial\left(\pi_{n}\left(\mathbb{D}^{n}\right)\right)$. Here $\mathbb{D}^{n}$ denotes the unit polydisc in $\mathbb{C}^{n}$ and $\partial \mathbb{D}^{n}=\partial \mathbb{D} \times \cdots \times \partial \mathbb{D}$. We refer to [19] and [27] for information about proper holomorphic mappings. Let now $\mathbb{G}_{n}=\pi_{n}\left(\mathbb{D}^{n}\right)$. The domain $\mathbb{G}_{n}$ is called the symmetrized polydisc. In [16] it was proved there exists a plurisubharmonic function $\varphi: \Omega \rightarrow(-\infty, 0)$ such that the closure of the set $\{z \in \Omega: \varphi(z)<c\}$ is compact in $\Omega$ for every $c \in(-\infty, 0)$, i.e., $\mathbb{G}_{n}$ is a hyperconvex domain. It is worth mentioning that $\mathbb{G}_{2}$ is the first non-trivial example of a domain on which the Lempert function, the Kobayashi distance and the Charathéodory distance coincide and which is not exhausted by domains biholomorphic to convex domains (see e.g. [1], [12], [13], [15] and [16]).

Let $I$ be an increasing multi-index, $1 \leq i_{1}<\cdots<i_{k} \leq n$; we will write $i \in I$ to mean that $i \in\left\{i_{1}, \ldots, i_{k}\right\}$. Let $\delta_{I}$ denote the set

$\pi_{n}\left(\mathbb{D}_{1} \times \cdots \times \mathbb{D}_{i_{1}-1} \times \partial \mathbb{D}_{i_{1}} \times \mathbb{D}_{i_{1}+1} \times \cdots \times \mathbb{D}_{i_{k}-1} \times \partial \mathbb{D}_{i_{k}} \times \mathbb{D}_{i_{k}+1} \times \cdots \times \mathbb{D}_{n}\right)$, where the index only refers to the position. Then $\delta_{I}=\delta_{J}$ if $I$ and $J$ are of the same length. This implies that

$$
\partial \mathbb{G}_{n}=\bigcup_{k=1}^{n} \pi_{n}(\underbrace{\partial \mathbb{D} \times \cdots \times \partial \mathbb{D}}_{k \text { times }} \times \underbrace{\mathbb{D} \times \cdots \times \mathbb{D}}_{n-k \text { times }})=: \bigcup_{k=1}^{n} \delta_{k} .
$$


Note that it was proved in [16] that $\delta_{n}=\pi_{n}\left((\partial \mathbb{D})^{n}\right)$ is the Shilov boundary of $\mathbb{G}_{n}$. We are now in a position to define plurisubharmonic and pluriharmonic functions on $\partial \mathbb{G}_{n}$ (see Definition 3.1 for the case when the domain of definition is the boundary of a hyperconvex product domain).

Definition 5.1. An upper semicontinuous function $u: \partial \mathbb{G}_{n} \rightarrow \mathbb{R} \cup$ $\{-\infty\}$ is plurisubharmonic if $u$ is plurisubharmonic on every $\delta_{k}$ for $k \in$ $\{1, \ldots, n-1\}$, i.e., for all $k \in\{1, \ldots, n-1\}$ and $w_{1}, \ldots, w_{k} \in \partial \mathbb{D}$, the function defined by

$$
\left(z_{1}, \ldots, z_{n-k}\right) \mapsto u \circ \pi_{n}\left(w_{1}, \ldots, w_{k}, z_{1}, \ldots, z_{n-k}\right)
$$

is plurisubharmonic on $\mathbb{D}^{n-k}$. The identically $-\infty$ function is not considered plurisubharmonic. In a similar manner a continuous function $u: \partial \mathbb{G}_{n} \rightarrow \mathbb{R}$ is pluriharmonic if $u$ is pluriharmonic on every $\delta_{k}$ for $k \in\{1, \ldots, n-1\}$.

The following property of the symmetrized polydisc proved by Edigarian and $\mathrm{Z}$ wonek is an important tool in the proof of our main theorem.

Proposition 5.2. Let $A$ be a domain in $\mathbb{C}^{m}, m \geq 1$, and let $\varphi: A \rightarrow \mathbb{C}^{n}$ be a holomorphic function. Then the following holds:

(a) if $\varphi(A) \subseteq \delta_{n}$, then $\varphi$ is constant,

(b) if $\varphi(A) \subseteq \partial \mathbb{G}_{n}$, then there exists a $k, 1 \leq k \leq n$, such that $\varphi(A) \subseteq \delta_{k}$.

Proof. See Lemmas 4 and 5 in [16].

Lemma 5.3. Let $n \geq 2$, and let $f: \partial \mathbb{G}_{n} \rightarrow \mathbb{R}$ be a continuous function. The following conditions are then equivalent:

(1) there exists $u \in \mathcal{P S H}\left(\mathbb{G}_{n}\right) \cap C\left(\overline{\mathbb{G}}_{n}\right)$ such that $\left.u\right|_{\partial \mathbb{G}_{n}}=f$,

(2) $f$ is plurisubharmonic in the sense of Definition 5.1,

(3) $f$ is subharmonic on every analytic disc d embedded in $\partial \mathbb{G}_{n}$, i.e., $f \circ d$ is subharmonic on $\mathbb{D}$.

Proof. First note that the equivalence of (2) and (3) follows immediately from Proposition 5.2 and Definition 5.1. We next prove $(3) \Rightarrow(1)$ : first, we define $g=f \circ \pi_{n}: \partial \mathbb{D}^{n} \rightarrow \mathbb{R}$. This is a continuous function on $\partial \mathbb{D}^{n}$. By assumption (3), $g$ is subharmonic on every analytic disc $d$ embedded in $\partial \mathbb{D}^{n}$. Theorem 4.2 implies that there exists a function $s$ which is pluriharmonic on $\mathbb{D}^{n}$, continuous on $\overline{\mathbb{D}}^{n}$ and $\left.s\right|_{\partial \mathbb{D}^{n}}=g$. Let $\varphi: \mathbb{G}_{n} \rightarrow \mathbb{R}$ be defined by

$$
\varphi(w)=\max \left\{s(z): z \in \pi_{n}^{-1}(w)\right\} .
$$

From [20] it follows that $\varphi$ is plurisubharmonic on $\mathbb{G}_{n}$ and $\left.\varphi\right|_{\partial \mathbb{G}_{n}}=f$ by construction. Walsh's theorem (see [29]) implies that $P B_{f} \in \mathcal{P S H}\left(\mathbb{G}_{n}\right) \cap$ $C\left(\overline{\mathbb{G}}_{n}\right)$. Hence, (1) follows by letting $u=P B_{f}$.

The final step is to prove $(1) \Rightarrow(2)$. Let $f_{w_{1}, \ldots, w_{k}}: \mathbb{D}^{n-k} \rightarrow \mathbb{R} \cup\{-\infty\}$ be defined as in (5.1); we need to prove that this function is plurisubharmonic under the assumption that there exists $u \in \mathcal{P S H}\left(\mathbb{G}_{n}\right) \cap C\left(\overline{\mathbb{G}}_{n}\right)$ such that 
$\left.u\right|_{\partial \mathbb{G}_{n}}=f$. Let $k \in\{1, \ldots, n-1\}, w_{1}, \ldots, w_{k} \in \partial \mathbb{D}$ and take a sequence $\left[\left(w_{1}^{m}, \ldots, w_{k}^{m}\right)\right]_{m=1}^{\infty}$ in $\mathbb{D}^{n-k}$ which converges to $\left(w_{1}, \ldots, w_{k}\right)$ as $m \rightarrow \infty$. Moreover, let $\left[u_{m}\right]$ be the sequence of real-valued function on $\mathbb{D}^{n-k}$ defined by

$$
u_{m}\left(z_{1}, \ldots, z_{n-k}\right)=u \circ \pi_{n}\left(w_{1}^{m}, \ldots, w_{k}^{m}, z_{1}, \ldots, z_{n-k}\right) .
$$

Then $u_{m}$ is pluriharmonic on $\mathbb{D}^{n-k}$, and continuous up to the boundary. The sequence $\left[u_{m}\right]$ converges uniformly to $f_{w_{1}, \ldots, w_{k}}$ on $\mathbb{D}^{n-k}$ as $m \rightarrow \infty$, and therefore $f$ is plurisubharmonic in the sense of Definition 5.1.

Theorem 5.4. Let $n \geq 3$. If $f: \partial \mathbb{G}_{n} \rightarrow \mathbb{R}$ is continuous, then the following assertions are equivalent:

(1) there exists a function $u$ which is pluriharmonic on $\mathbb{G}_{n}$, continuous on $\overline{\mathbb{G}}_{n}$ and $\left.u\right|_{\partial \mathbb{G}_{n}}=f$,

(2) $f$ is pluriharmonic on $\partial \mathbb{G}_{n}$ in the sense of Definition 5.1,

(3) $f$ is harmonic on every analytic disc d embedded in $\partial \mathbb{G}_{n}$, i.e., $f \circ d$ is harmonic on $\mathbb{D}$ for every injective, holomorphic function $d: \mathbb{D} \rightarrow \overline{\mathbb{G}}_{n}$ with $d(\mathbb{D}) \subseteq \partial \mathbb{G}_{n}$,

(4) the Perron-Bremermann envelope $P B_{f}$ is pluriharmonic on $\mathbb{G}_{n}$, i.e.,

$$
P B_{-f}=-P B_{f}
$$

(5) for every $z_{0} \in \partial \mathbb{G}_{n}$ and every Jensen measure $\mu$ with barycenter $z_{0}$,

$$
f\left(z_{0}\right)=\int_{\partial \mathbb{G}_{n}} f d \mu
$$

(6) $f$ is compliant on $\partial \mathbb{G}_{n}$.

Proof. The equivalence of (1), (2) and (3) follows immediately from Lemma 5.3, and $(5) \Leftrightarrow(6)$ and $(4) \Rightarrow(5)$ follow from Theorem 2.4 .

To prove $(5) \Rightarrow(2)$, let $k \in\{1, \ldots, n-1\}$ and $z_{0} \in \delta_{k}$. Take any complex line $l$ through $z_{0}$, and $r>0$ such that $z_{0}+r \mathbb{D} \subset l \cap \delta_{k}$. Since the Lebesgue measure $\lambda$ on $\mathbb{D}$ is a Jensen measure at $z_{0}$ we have by assumption

$$
f\left(z_{0}\right)=\int_{z_{0}+r \mathbb{D}} f d \lambda
$$

which implies that $f$ is harmonic at $z_{0}$ and therefore $f$ is pluriharmonic on $\partial \mathbb{G}_{n}$.

To complete the proof we need to prove that (1), (2) and (3) imply (4). We proceed as in the proof of Lemma 5.3 by defining $g=f \circ \pi_{n}: \partial \mathbb{D}^{n} \rightarrow \mathbb{R}$. Then $g$ is pluriharmonic on $\partial \mathbb{D}^{n}$ and therefore $P B_{g}$ is pluriharmonic on $\mathbb{D}^{n}$ and continuous on $\overline{\mathbb{D}}^{n}$ by Theorem 3.3. Define

$$
\varphi(w)=\max \left\{P B_{g}(z): z \in \pi_{n}^{-1}(w)\right\} .
$$

From [20] it follows that $\varphi \in \mathcal{P S H}\left(\mathbb{G}_{n}\right) \cap C\left(\overline{\mathbb{G}}_{n}\right)$ and $\left.\varphi\right|_{\partial \mathbb{G}_{n}}=f$, hence $\varphi \leq P B_{f} \in \mathcal{P S H}\left(\mathbb{G}_{n}\right) \cap C\left(\overline{\mathbb{G}}_{n}\right)$. Therefore $P B_{f} \circ \pi_{n} \in \mathcal{P S H}\left(\mathbb{D}^{n}\right)$ and 
$\left.\left(P B_{f} \circ \pi_{n}\right)\right|_{\partial \mathbb{D}^{n}}=g$. Thus, for $z \in \pi_{n}^{-1}(w)$ we get $\left(P B_{f} \circ \pi_{n}\right)(z) \leq P B_{g}(z)$ and therefore $P B_{f}(w) \leq \varphi(z)$, which implies that $\varphi=P B_{f}$.

Next we prove that $P B_{f}=-P B_{-f}$. Note that $P B_{g}=-P B_{-g}$, since $P B_{g}$ is pluriharmonic on $\mathbb{D}^{n}$ and continuous on $\overline{\mathbb{D}}^{n}$ and

$$
\begin{aligned}
P B_{f}(w) & =\max \left\{P B_{g}(z): z \in \pi_{n}^{-1}(w)\right\} \\
& =\max \left\{-P B_{-g}(z): z \in \pi_{n}^{-1}(w)\right\} \\
& =-\min \left\{P B_{-g}(z): z \in \pi_{n}^{-1}(w)\right\} .
\end{aligned}
$$

In a similar manner we get $P B_{-f}(w)=\max \left\{P B_{-g}(z): z \in \pi_{n}^{-1}(w)\right\}$. Combining these two representations we obtain

$$
\begin{aligned}
0 \geq P B_{f}(w)+P B_{-f}(w)= & \max \left\{P B_{-g}(z): z \in \pi_{n}^{-1}(w)\right\} \\
& -\min \left\{P B_{-g}(z): z \in \pi_{n}^{-1}(w)\right\} \geq 0,
\end{aligned}
$$

which shows that $P B_{f}=-P B_{-f}$ and completes the proof.

By using Example 3.4 it is possible to construct a continuous function $f: \partial \mathbb{G}_{2} \rightarrow \mathbb{R}$ such that (2) of Theorem 5.4 holds but not (1); and by using Example 3.7 it is possible to show that the implication $(6) \Rightarrow(4)$ is, in general, not true for $n=2$. If $f: \partial \mathbb{G}_{2} \rightarrow \mathbb{R}$ is a pluriharmonic function in the sense of Definition 5.1, then

$$
\int_{\mathbb{D}^{2}}\left(d d^{c}\left(P B_{f \circ \pi_{2}}+P B_{-f \circ \pi_{2}}\right)\right)^{2}=2 \int_{\mathbb{G}_{2}}\left(d d^{c}\left(P B_{f}+P B_{-f}\right)\right)^{2},
$$

and by Theorem 4.2 for every $(\zeta, \xi) \in \partial \mathbb{G}_{2}$ we have

$$
\lim _{\substack{(z, w) \rightarrow(\zeta, \xi) \\(z, w) \in \mathbb{G}_{2}}}\left(P B_{f}+P B_{-f}\right)(z, w)=0 .
$$

By using Proposition 3.8, this yields

Proposition 5.5. If $f: \partial \mathbb{G}_{2} \rightarrow \mathbb{R}$ is a pluriharmonic function in the sense of Definition 5.1 which satisfies

$$
\sum_{k_{1}, k_{2}=0}^{\infty} \sqrt{k_{1} k_{2}}\left|b_{k_{1}, k_{2}}\right|<\infty,
$$

then $f$ is compliant on $\mathbb{G}_{2}$. Here

$$
b_{k_{1}, k_{2}}=\int_{\partial \mathbb{D} \times \partial \mathbb{D}} w_{1}^{k_{1}} \bar{w}_{2}^{k_{2}} f\left(w_{1}+w_{2}, w_{1} w_{2}\right) d \sigma\left(w_{1}\right) d \sigma\left(w_{2}\right),
$$

where $d \sigma$ is the normalized Lebesgue measure on $\partial \mathbb{D}$.

In [26], Rudin characterizes those real-valued continuous functions on $\partial \mathbb{D}^{2}$ which can be extended to a pluriharmonic function on $\mathbb{D}^{2}$. Using Rudin's result we obtain a similar result for $\partial \mathbb{G}_{2}$. 
Proposition 5.6. Let $f: \partial \mathbb{G}_{2} \rightarrow \mathbb{R}$ be continuous. The following are then equivalent:

(1) there exists a function $u$ which is pluriharmonic on $\mathbb{G}_{2}$, continuous on $\overline{\mathbb{G}}_{2}$ and $\left.u\right|_{\partial \mathbb{G}_{2}}=f$,

(2) $f$ satisfies $b_{k_{1}, k_{2}}=0$ for any $k_{1}, k_{2} \in \mathbb{N}$, where $b_{k_{1}, k_{2}}$ is defined as in Proposition 5.5.

Proof. $(1) \Rightarrow(2)$ : By assumption, $P B_{f}$ is pluriharmonic on $\mathbb{G}_{2}$ and therefore $P B_{f \circ \pi_{2}}$ is pluriharmonic on $\mathbb{D}^{2}$, since $P B_{f \circ \pi_{2}}=\left(P B_{f}\right) \circ \pi_{2}$. Rudin's characterization in [26] yields

$$
\int_{\partial \mathbb{D} \times \partial \mathbb{D}} w_{1}^{k_{1}} \bar{w}_{2}^{k_{2}} f \circ \pi_{2}\left(w_{1}, w_{2}\right) d \sigma\left(w_{1}\right) d \sigma\left(w_{2}\right)=0
$$

for any $k_{1}, k_{2} \in \mathbb{N}$, and therefore (2) holds.

$(2) \Rightarrow(1)$ : Let $f$ satisfy condition (2). Then (5.2) holds for any $k_{1}, k_{2} \in \mathbb{N}$. Rudin's characterization again shows that $P B_{f \circ \pi_{2}}$ is pluriharmonic on $\mathbb{D}^{2}$ and therefore

$$
0=\int_{\mathbb{D}^{2}}\left(d d^{c}\left(P B_{f \circ \pi_{2}}+P B_{-f \circ \pi_{2}}\right)\right)^{2}=2 \int_{\mathbb{G}_{2}}\left(d d^{c}\left(P B_{f}+P B_{-f}\right)\right)^{2} .
$$

Hence, $\left(d d^{c}\left(P B_{f}+P B_{-f}\right)\right)^{2}=0$ on $\mathbb{G}_{2}$, which implies that $P B_{f}+P B_{-f}=0$ on $\mathbb{G}_{2}$ (see e.g. [7]). Thus $P B_{f}$ is the desired function and the proof is complete.

\section{References}

[1] J. Agler and N. J. Young, The hyperbolic geometry of the symmetrized bidisc, J. Geom. Anal. 14 (2004), 375-403.

[2] P. Åhag and R. Czyż, The connection between the Cegrell classes and compliant functions, Math. Scand. 99 (2006), 87-98.

[3] D. H. Armitage and S. J. Gardiner, Classical Potential Theory, Springer Monogr. Math., Springer, 2001.

[4] E. Bedford and P. Federbush, Pluriharmonic boundary values, Tôhoku Math. J. 26 (1974), 505-511.

[5] H. Begehr and A. Dzhuraev, An Introduction to Several Complex Variables and Partial Differential Equations, Pitman Monogr. Surveys Pure Appl. Math. 88, Longman, 1997.

[6] - - - Schwarz problem for Cauchy-Riemann systems in several complex variables, in: Analysis and Topology, World Sci., River Edge, 1998, 63-114.

[7] Z. Błocki, On the $L^{p}$ stability for the complex Monge-Ampère operator, Michigan Math. J. 42 (1995), 269-275.

[8] - Interior regularity of the complex Monge-Ampère equation in convex domains, Duke Math. J. 105 (2000), 167-181.

[9] M. Carlehed, U. Cegrell and F. Wikström, Jensen measures, hyperconvexity and boundary behaviour of the pluricomplex Green function, Ann. Polon. Math. 71 (1999), 87-103. 
[10] U. Cegrell, Pluricomplex energy, Acta Math. 180 (1998), 187-217.

[11] - , The general definition of the complex Monge-Ampère operator, Ann. Inst. Fourier (Grenoble) 54 (2004), 159-179.

[12] C. Costara, Le problème de Nevanlinna-Pick spectral, Ph.D. Thesis, Laval Univ., 2004.

[13] —, The symmetrized bidisc and Lempert's theorem, Bull. London Math. Soc. 36 (2004), 656-662.

[14] A. Dzhuraev, On Riemann-Hilbert boundary problem in several complex variables, Complex Variables Theory Appl. 29 (1996), 287-303.

[15] A. Edigarian, A note on C. Costara's paper: "The symmetrized bidisc and Lempert's theorem", Ann. Polon. Math. 83 (2004), 189-191.

[16] A. Edigarian and W. Zwonek, Geometry of the symmetrized polydisc, Arch. Math. (Basel) 84 (2005), 364-374.

[17] G. Fichera, Problemi al contorno per le funzioni pluriarmoniche, in: Proc. Conf. in Honor of the 80th Anniversary of the Birth of Renato Calapso (Messina/Taormina, 1981), Veschi, Rome, 1981, 127-152.

[18] J.-E. Fornæss and J. Wiegerinck, Approximation of plurisubharmonic functions, Ark. Mat. 27 (1989), 257-272.

[19] F. Forstnerič, Proper holomorphic mappings: a survey, in: Several Complex Variables (Stockholm, 1987/1988), Math. Notes 38, Princeton Univ. Press, Princeton, NJ, 1993, 297-363.

[20] M. Klimek, On the invariance of the L-regularity under holomorphic mappings, Zeszyty Nauk. Uniw. Jagielloń. Prace Mat. 23 (1982), 27-38.

[21] S. Kołodziej, The complex Monge-Ampère equation and pluripotential theory, Mem. Amer. Math. Soc. 178 (2005).

[22] A. Perotti, Dirichlet problem for pluriharmonic functions of several complex variables, Comm. Partial Differential Equations 24 (1999), 707-717.

[23] - , Tangential form of the trace condition for pluriharmonic functions in $\mathbb{C}^{n}$, Complex Variables Theory Appl. 48 (2003), 615-623.

[24] H. Poincaré, Sur les fonctions de deux variables, Acta Math. 2 (1883), 97-113.

[25] E. A. Poletsky, personal communication, Szczyrk, 2006.

[26] W. Rudin, Function Theory in Polydiscs, Benjamin, 1969.

[27] - Proper holomorphic maps and finite reflection groups, Indiana Univ. Math. J. 31 (1982), 701-720.

[28] A. Sadullaev, Solution of the Dirichlet problem in a polydisc for the complex MongeAmpère equation, Dokl. Akad. Nauk SSSR 267 (1982), 563-566 (in Russian); English transl.: Soviet Math. Dokl. 26 (1982), 671-674.

[29] J. B. Walsh, Continuity of envelopes of plurisubharmonic functions, J. Math. Mech. 18 (1968), 143-148.

[30] F. Wikström, Jensen measures and boundary values of plurisubharmonic functions, Ark. Mat. 39 (2001), 181-200.

Mid Sweden University

SE-851 70 Sundsvall, Sweden

E-mail: per.ahag@miun.se
Institute of Mathematics Jagiellonian University Reymonta 4

30-059 Kraków, Poland

E-mail: Rafal.Czyz@im.uj.edu.pl 